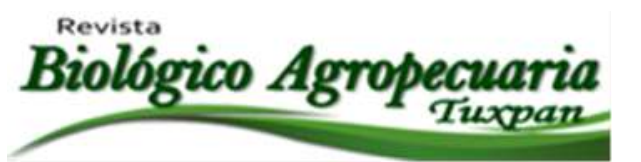

\title{
Estilos de vida en estudiantes de posgrado de una universidad pública
}

Lifestyles and nutrition in postgraduate students of a public university

Báez Palmeros Marycruz ${ }^{1}$, Claudia Beatriz Enríquez Hernández ${ }^{1 凶}$, Edith Castellanos Contreras ${ }^{1}$, María Esther Fragoso Terán ${ }^{2}$, Javier Salazar Mendoza ${ }^{3}$

${ }^{1}$ Facultad de Enfermería de la Universidad Veracruzana, Región Veracruz, Ver, México. ${ }^{2}$ Facultad de Contaduría de la Universidad Veracruzana, Región Veracruz, México. ${ }^{3}$ Facultad de Enfermería de la Universidad Veracruzana, Región Córdoba-Orizaba, México.

$\bowtie$ Autor para correspondencia: beenriquez@uv.mx

Recibido: 15/04/2019

Aceptado: 15/05/2019

\section{RESUMEN}

El estilo de vida saludable de las personas es un constructo multidimensional que involucra una cadena de factores psicológicos, sociales, culturales y económicos que tienden a mantener un estado de salud física y mental. Esto resulta de gran importancia, ya que permite definir a las personas y ubicarlas en categorías en función de la manera en que viven y desarrollan su conducta. Objetivo: Describir los estilos de vida de los estudiantes del Programa de Posgrado Maestría en Enfermería. Material y Métodos: Estudio cuantitativo, de tipo descriptivo-transversal, con una muestra de 14 participantes. Se aplicó el cuestionario de Percepción de Salud (PEPS-I) de Nola J. Pender, el cual está conformado por 48 ítems repartidos en 6 dimensiones: Nutrición, ejercicio, responsabilidad en salud, manejo del estrés, soporte interpersonal y auto actualización. Resultados: El 7,1 \% de los estudiantes tiene un estilo de vida saludable y el 92,9 \% puntuaron un estilo de vida no saludable. Conclusiones: Se obtuvo una puntuación no favorecedora para los estilos de vida de los estudiantes de posgrado, ya que de acuerdo a Pender (2015) menciona que para considerar un estilo de vida este debe puntuar por encima de la media en cada dimensión, concluyendo así que los estudiantes del Programa de Posgrado Maestría no tienen estilos de vida saludable.

Palabras claves: Estilos de vida, Estudiantes de posgrado, enfermería.

\begin{abstract}
ABSTRAC
The healthy lifestyle of people is a multidimensional construct that involves a chain of psychological, social, cultural and economic factors that tend to maintain a state of physical and mental health. This is of great importance, since it allows defining people and placing them in categories according to the way they live and develop their behavior. Objective: Describe the lifestyles of the students of the Master's Program in Nursing. Materials \& Methods: Quantitative research, descriptive-transversal type, with a sample of 14 participants. The Perception of Health questionnaire (PEPS-I) was applied by Nola J. Pender, which is made up of 48 items divided into 6 dimensions: Nutrition, exercise, health responsibility, stress management, interpersonal support and self-actualization. Results: $7.1 \%$ of the students have a healthy lifestyle and $92.9 \%$ rated an unhealthy lifestyle. Conclusions: A non-favorable score was obtained for the lifestyles of postgraduate students, since according to Pender (2015) it


mentions that to consider a lifestyle this must score above the average in each dimension, thus concluding that students of the Graduate Master's Program do not have healthy lifestyles.

Keywords: Lifestyles, Graduate students, nursing.

\section{INTRODUCCIÓN}

La Organización Mundial de la Salud (OMS, 1998), define al estilo de vida como aquella forma de vida basada en determinados patrones de conductas, hábitos y/o costumbres que condicionan las características propias de la persona, las relaciones interpersonales $y$ condiciones economicas. Este conjunto de patrones conductuales que la persona pone en práctica cotidianamente, puede ser pertinente para el mantenimiento de su salud, o que lo pueden llegar a colocar en una situación de riesgo. El estilo de vida saludable de las personas o de los grupos es un constructo multidimensional que involucra una cadena de factores psicológicos, sociales, culturales y hasta económicos que tienen a mantener un adecuado estado de salud física y mental. Esto resulta de gran importancia, ya que permiten definir a las personas y ubicarlas en categorías en función de la manera en que viven y desarrollan su conducta (Maya, 2001; Oblitas, 2010).

A su vez, es un proceso social que involucra una serie de tradiciones, hábitos y conductas de los individuos y grupos que conllevan la satisfacción de necesidades humanas para alcanzar el bienestar y una vida más satisfactoria. Estas conductas, hábitos y tradiciones son trasmitidos al individuo por el núcleo familiar, y se originan a través de la percepción de salud de esta y de las situaciones que son presentadas cotidianamente. Reflejando el estilo de vida en diferentes dimensiones como responsabilidad en salud, actividad física, nutrición, relaciones interpersonales, crecimiento personal y tratamiento del estrés. Las prácticas de conductas patógenas, tiene consecuencias negativas en la salud de las personas, que puede derivar en complicaciones, además de estados emocionales negativos, derivados del estrés. Un factor determinante para el estilo de vida de las personas en la actualidad es su ritmo de vida (Infiesta, Bimella, Garrucho \& March, 2004; Oblitas, 2010)

El estilo de vida da como resultado los hábitos que una persona asumirá para su nutrición y actividad física, esto como resultado, determinará si su alimentación será saludable, equilibrada, variada y su ejercicio será el requerido para el consumo de energía diaria, así como, la localización de su adiposidad, si es cintura o cadera, ya que todos estos factores pueden ser desencadenantes de enfermedades crónico degenerativas. Por lo cual se llega al objetivo; describir cuales son los estilos de vida de los estudiantes del Programa de Posgrado Maestría en Enfermería.

\section{MATERIALES Y MÉTODOS}

La metodología utilizada fue de diseño cuantitativo de nivel descriptiva-transversal, el diseño se considera apto para este estudio ya que describe la variable. La población se conformó por estudiantes de la Maestría en Enfermería. La muestra fue de 14 participantes de ambos sexos inscritos a la Maestría en Enfermería, donde se aplicó el instrumento Cuestionario de Perfil de Estilo de Vida de Nola J. Pender a los 
participantes que firmaron el consentimiento informado.

Para el análisis de los datos se utilizó el programa estadístico informatico Statistical Package for the Social Sciences (SPSS), versión 23. Se empleó una codificación de datos para la base de datos de acuerdo a las dimensiones del instrumento para su tabulación. Para la presentación de los resultados se utilizaron tablas de frecuencia y porcentaje de acuerdo a la naturaleza de los datos obtenidos.

El instrumento aplicado para la recolección de datos constaba de un consentimiento informado donde se exponía grosso modo sobre el objetivo metodología de la investigación, así como las considerciones éticas. Se aplicó el Cuestionario de Perfil de Estilo de Vida Saludable versión I (PEPS-I) de Nola J. Pender, traducido al español. Este instrumento permite medir el estilo de vida promotor de la salud. Está conformado por 48 preguntas, divididas en 6 dimensiones: Nutrición, ejercicio, responsabilidad en salud, manejo del estrés, soporte interpersonal y autoactualización, que le corresponden los reactivos a nutrición $1,5,14,19,26,35$; a ejercicio 4, 13, 22, 30, 38; a responsabilidad en salud 2, 7, 15, 20, 28, 32, 33, 42, 43, 46; a manejo de estrés $6,11,27,36,40,41,45$; a soporte interpersonal 10, 18, 24, 25, 31, 39, 47, y a Autoactualización 3, 8, 9, 12, 16, 17, 21, 23, 29, $34,37,44,48$.

Este cuestionario tiene un patrón de respuestas tipo likert de cuatro criterios ( $1=$ nunca; $2=\mathrm{a}$ veces; 3 = frecuentemente; $4=$ rutinariamente); el valor total que toma este cuestionario va de 48 a 192 puntos, a mayor puntuación, mejor estilo de vida. Instrumento validado con un coeficiente alfa de Cronbach de .89 para el índice de EV General.
Se tomaron medidas antropométricas; peso corporal, talla, circunferencia de cintura y cadera, dichas medidas fueron registradas en una cédula cada una en el número de folio correspondiente al participante. Para el peso corporal y la talla, se utilizó una báscula con estadimetro con capacidad de peso de hasta 160 $\mathrm{kg}$, y de talla de $210 \mathrm{~cm}$, se colocó sobre una superficie plana, horizontal y firme.

La medición del peso se realizó con la menor ropa posible y sin zapatos, se le pidió al participante que subiera a la báscula colocando los pies paralelos en el centro, de frente al investigador. Se le explicó que debía estar erguido, con la vista hacia el frente, sin moverse y con los brazos naturalmente relajados a los lados, se tomó la lectura cuando la aguja central se encontró en medio de los dos márgenes sin moverse.

Se prosiguió a realizar la medición de la talla, se le explicó al participante la forma correcta de la posición de los pies, que es, con los talones juntos y las puntas de los pies ligeramente separados y centrados en medio de la base del estadimetro. Para la lectura se trazó una línea imaginaria del orificio del oído a la base de la órbita del ojo. Esta línea tenía que estar paralela a la base del estadimetro y formar un Angulo recto con respecto a la pared y se anotó el valor más próximo (Secretaria de Salud, 2002).

La circunferencia de cintura y cadera fueron tomadas con una cinta métrica, para la medición se trazó una línea imaginaria que partía del hueco de la axila hasta la cresta iliaca. Sobre esta se identificó la cintura en el punto medio entre la última costilla y la parte superior de la cresta iliaca o cadera. Se colocó la cinta métrica sobre estos dos puntos y se tomó el registro (Secretaria de Salud, 2002). 


\section{RESULTADOS}

Se puede notar en la Tabla $\mathrm{N}^{\circ} 1$, que el $85,7 \%$ de los participantes son mujeres y el $14,3 \%$ hombres. En cuanto a la edad el 50\% refirió tener
26 años. Por otro lado en el estado civil el 92,9\% de los participantes son solteros. El 78,6\% se confirmó ser foráneos.

Tabla 1.Datos generales de la población.

\begin{tabular}{|c|c|c|c|}
\hline & Varible & $\mathrm{fr}$ & $\%$ \\
\hline \multirow{2}{*}{ Sexo } & Masculino & 2 & 14,3 \\
\hline & Femenino & 12 & 85,7 \\
\hline \multirow{5}{*}{ Edad } & 24 & 3 & 21,4 \\
\hline & 25 & 1 & 7,1 \\
\hline & 26 & 7 & 50,0 \\
\hline & 27 & 2 & 14,3 \\
\hline & 28 & 1 & 7,1 \\
\hline \multirow{2}{*}{ Estado civil } & Soltero & 13 & 92,9 \\
\hline & Casado & 1 & 7,1 \\
\hline \multirow{2}{*}{$\begin{array}{l}\text { Lugar } \\
\text { residencia }\end{array}$} & Residente de Veracruz & 3 & 21,4 \\
\hline & Foráneo & 11 & 78,6 \\
\hline \multirow{2}{*}{ Vive } & Solo & 3 & 21,4 \\
\hline & Con familia & 11 & 78,6 \\
\hline \multirow{2}{*}{ Su casa es } & Rentada & 4 & 28,6 \\
\hline & Propia & 10 & 71,4 \\
\hline Ingreso mensual & Más de $\$ 8,000$ & 14 & 100 \\
\hline
\end{tabular}

Fuente: Directa $(\mathrm{n}=14)$.

En la Tabla 2, se llegó a la conclusión de considerar la clasificación como estilo de vida saludable, y estilo de vida no saludable. De esta forma, se pudo determinar solo 1 caso de un estilo de vida saludable, y 13 casos con un estilo de vida no saludable.

Tabla 2. Clasificación del estilo de vida

\begin{tabular}{lll}
\hline Indicadores & Fr & $\%$ \\
\hline Estilo de vida saludable & 1 & 7,1 \\
Estilo de vida no saludable & 13 & 92,9 \\
Total & 14 & 100 \\
\hline
\end{tabular}

Fuente: Directa $(\mathrm{n}=14)$.

En la Tabla 3 se puede observar la clasificación de estilos de vida por dimensiones donde el
92,9\% de los estudiantes presentan una conducta no saludable con respecto al manejo de estrés y 
Báez et al., 2019

responsabilidad en la salud. Todas las dimensiones valoradas en esta tabla presentan conductas negativas pero el $28,6 \%$ tienen más interés en el ejercicio como conducta saludable.

Tabla 3. Clasificación por dimensiones del Cuestionario de Perfil de Estilos de Vida

\begin{tabular}{|c|c|c|c|}
\hline Dimensión & Indicadores & fr & $\%$ \\
\hline \multirow{2}{*}{ Nutrición } & Conductas Saludables & 2 & 14,3 \\
\hline & Conductas No Saludables & 12 & 85,7 \\
\hline \multirow{2}{*}{ Ejercicio } & Conductas Saludables & 4 & 28,6 \\
\hline & Conductas No Saludables & 10 & 71,4 \\
\hline \multirow{2}{*}{$\begin{array}{l}\text { Responsabilidad en } \\
\text { salud }\end{array}$} & Conductas Saludables & 1 & 7,1 \\
\hline & Conductas No Saludables & 13 & 92,9 \\
\hline \multirow{2}{*}{ Manejo del estrés } & Conductas Saludables & 1 & 7,1 \\
\hline & Conductas No Saludables & 13 & 92,9 \\
\hline \multirow{2}{*}{ Soporte interpersonal } & Conductas Saludables & 2 & 14,3 \\
\hline & Conductas No Saludables & 12 & 85,7 \\
\hline \multirow{2}{*}{ Autoactualización } & Conductas Saludables & 2 & 14,3 \\
\hline & Conductas No Saludables & 12 & 85,7 \\
\hline
\end{tabular}

Fuente: Directa $(\mathrm{n}=14)$

En la clasificación del índice de masa corporal podemos observar en la tabla 4 que el $57,1 \%$ de los estudiantes cuentan con un peso normal, mientras que el $14,3 \%$ presentan sobrepeso y obesidad tipo I.

Tabla 4. Clasificación de acuerdo al Índice de masa corporal

\begin{tabular}{lll}
\hline Indicador nutricional & fr & $\%$ \\
\hline Bajo peso & 1 & 7,1 \\
peso normal & 8 & 57,1 \\
Sobrepeso & 2 & 14,3 \\
obesidad tipo I & 2 & 14,3 \\
obesidad tipo II & 1 & 7,1 \\
Total & 14 & 100,0 \\
\hline
\end{tabular}

Fuente: Directa $(n=14)$ 
En la Tabla 5 de clasificación general del cociente cintura/cadera se puede notar que el $78,6 \%$ del total de los estudiantes, presentan adiposidad en la zona del abdomen, solo el $21,4 \%$ presentan un cociente normal.

Tabla 5. Clasificación general del cociente cintura/cadera

\begin{tabular}{lll}
\hline Indicador & fr & $\%$ \\
\hline Cociente normal & 3 & 21,4 \\
Adiposidad abdominal & 11 & 78,6 \\
Total & 14 & 100
\end{tabular}

Fuente: Directa $(n=14)$

Finalmente, en la Tabla 6 se observa que el caso $\mathrm{N}^{\circ} 4$ presenta una Conducta Saludable donde se ve reflejado en su estado nutricio, mientras que el resto de los estudiantes presentaron Conductas No Saludables y 6 de ellos presentan un estado nutricio no favorable.

Tabla 6. Estado nutricio y el estilo de vida

\begin{tabular}{lll}
\hline$N^{\circ}$ de caso & Estilo de vida & IMC \\
\hline 1 & Conducta No Saludable & Obesidad tipo II \\
2 & Conducta No Saludable & Sobrepeso \\
3 & Conducta No Saludable & Obesidad tipo I \\
4 & Conducta Saludable & Peso normal \\
5 & Conducta No Saludable & Peso normal \\
6 & Conducta No Saludable & Bajo peso \\
7 & Conducta No Saludable & Peso normal \\
8 & Conducta No Saludable & Peso normal \\
9 & Conducta No Saludable & Sobrepeso \\
10 & Conducta No Saludable & Obesidad tipo I \\
11 & Conducta No Saludable & Peso normal \\
12 & Conducta No Saludable & Peso normal \\
13 & Conducta No Saludable & Peso normal \\
14 & Conducta No Saludable & Peso normal \\
\hline
\end{tabular}

Fuente: Directa $(n=14)$ 


\section{DISCUSIÓN}

En el presente estudio, se encontró que el 7,1\% cuenta con un estilo de vida saludable y el 92,9 $\%$ presentaron estilos de vida no saludables de acuerdo a sus conductas que presentan en su vida diaria, siendo el ejercicio una de las conductas saludables adquirida con mayor frecuencia por los estudiantes representando solo el 28,6\%. Con base en este resultado, se encontró una similitud en el artículo "Nivel de estilo de vida de los alumnos universitarios de la escuela superior de Tlahuelilpan" realizado por Grimaldo (2012), en donde se halló un estilo de vida bueno en más del $25 \%$, un estilo de vida regular en más del 70 $\%$ y un estilo de vida malo en el 1,8\%. La similitud que podemos encontrar es que la mayoría de los estudiantes mantienen un estilo de vida regular, sin embargo, también podemos observar que hay más porcentaje de estudiantes con estilo de vida saludable, a este estudio.

En cuanto, a los resultados de las dimensiones se encontró una similitud en el artículo "Explorar el estilo de vida prevaleciente en estudiantes del área de la salud" de Peña (2009), se encontró que el mayor porcentaje de estudiantes presentaban conductas no saludables, en nutrición con más $60 \%$ y solo $14,8 \%$ practicaban conductas saludables, ejercicio con más del $60 \%$ y responsabilidad en salud con el 63,4\%.

\section{CONCLUSIONES}

Con los resultados obtenidos se llegó a la conclusión que la gran mayoría de los estudiantes presentaron conductas no saludables en su vida cotidiana, siendo muy pocos los interesados en la práctica de las actividades para la salud presentadas en las dimensiones del cuestionario de Perfil de Estilos de Vida Saludable, por lo cual el $92,9 \%$ presentan un estilo de vida no saludable. No obstante, en los resultados el cociente cintura/cadera, la mayoría mantiene adiposidad en el abdomen u obesidad androide, reflejo de las conductas no saludables que presentan estos estudiantes.

Como conducta a seguir se debe concientizar a los promotores de la salud a adquirir hábitos saludables para mejorar los estilos de vida que han presentado como estudiantes con el fin de favorecer la salud, el estado nutricio y asi evitar enfermedades crónico degenerativas, como las complicaciones que estas conlleven.

\section{LITERATURA CITADA}

Arboledas, G., \& Lluch, A. (2007). El sueño en el obeso. Importancia del cribado clinico. Acta Pedriatr Esp., 65(2), 74-78.

Arroyo, M., Rocandio, P., Ansotegui, L., Pascual, E., \& Rebato, E. (2006). Calidad de la dieta, sobrepeso y obesidad en estudiantes universitarios. Nutr Hosp, 21(6), 673-679.

Astiasarán, I. (2003). Alimentos y nutricion en la práctica sanitaria. Diaz de Santos.

Botica Artalejo, A. I. (2005). Servicios a la comunidad. Supuestos practicos practico. MAD-Eduforma.

Cegarra Sanches, J. (2011). Metodologia de la investigacion cientifica $\mathrm{y}$ tecnologica. Madrid: Ediciones

Grimaldo, M. M. (Enero-Junio de 2012). Estilo de vida saludable en estudiantes de posgrado de Ciencias de la Salud. Psicología y Salud, 22(1), 75-87.

Heyward, V. H. (2008). Evalución de la amplitud física y prescripción del ejercicio. Medica Panamericana.

Hoyos, G. P., Blanco, D. M., \& Sanchez, A. (Octubre - Diciembre de 2011). El modelo de promoción de la salud de Nola Pender. Una refexion en torno a su comprension. Medigraphic, 8(4). 
Infiesta, J., Bimella, J., Garrucho, G., \& March, J. (2004). Estilos de vida y juventud. ILO. Intra, M., Roales-Nieto, J., \& Moreno, E. (2011). Cambios en las conductas de riesgo y la salud en estudiantes universitarios argentinos a lo largo del periodo educativo. Rev int Psicol, 11(1), 139-147.

Leon Rubio, J. M. (2004). Psicología de la salud y de la calidad de vida. Barcelona: UOC.

Marinero Uribe, M. G. (2013). La gestión de la tutoría a nivel de posgrado. Diagnóstico de a práctica tutorial del 2009 al 2012, en una entidad académica de la Universidad Veracruzana. Tesis de Maestría en Ciencias Administrativas presentada al Instituto de Investigaciones y Estudios Superiores de las Ciencias Administrativas de la Universidad Veracruzana, Región Xalapa.

Ma-San, T. (2005). Guia para la promocion de estilos de vida saludables en educacion secundaria. Lima, Peru: Pixelstudio.

Matarese, L. E., \& Gottschlich, M. M. (2004). Nutrición clínica práctica. España: Elsevier Science.

Maya, L. (2001). Los estilos de vida saludables: componente de la calidad de vida. FUNLIBRE.

Morán, I., Cruz, V., \& Iñarritu, M. (2007). El indice de masa corporal y la imagen corporal percibida como indicadores del estado nutricional en universitarios. Rev Fac Med UNAM, 50(2), 76-79.

Moreno, E., Monereo Megías, S., \& Alvares Hernandez, J. (2000). Obesidad: Epidemia del siglo XXI. Madrid: Diaz de Santos.

Oblitas, L. A. (2010). Psicología de la salud y calidad de vida. Mexico: Cengage Learning Editores.

OMS. (1998). Promocion de la salud, Glosario.

OMS. (2015). Nota descriptiva $\mathrm{N}^{\circ} 394$ : Alimentacion sana. Obtenido de http://www.who.int/mediacentre/factsheet s/fs394/es/

OMS. (2016). Nota descriptiva $N^{\circ} 311$ : Obesidad y sobrepeso.

OMS. (2016). Nota descriptiva N³84: Actividad física.

Peña, L., Cano, A., Burguete, A., Castro, L., \& Leon, M. (2009). Efectos atribuibles a la procedencia de estudiantes universitarios sobre su estado nutricional: Foraneos y locales. Nutr Clin Diet Hop, 29(2), 40-45.

Ponce, G., Ruiz, E. j., Mañana, A., \& Arizona, B. (2011). Obesidad y factores de riesgo en estudiantes del area de la salud de la Universidad Autonoma de Baja California, Mexicali. RESPYN, 12(4), 115.

Ruiz Jiménez, M. A. (2004). Factores de riesgo cardiovascular en niños y adolescentes. Ediciones Diaz de Santos.

Sanabria-Ferrand, P., González, L., \& Urrego, D. (2007). Estilos de vida saludables en profesionales colombianos de la salud. Estudio exploratorio. Revista medica, 15(2), 207-217.

Secretaria de la Salud. (2007-2012). Programa de Accion Especifico 2007-2012: Promocion de la Salud: Una nueva cultura. México.

Secretaria de Salud. (2002). Toma de medidas clinicas y antropometricas en el adulto y adulto mayor. Manual de Procedimientos.

UNICEF. (2011). La desnutricion infantil. Punto\&coma.

Walker, S. N., \& Hill-Polerecky, D. M. (1996). Evaluación psicometrica del Perfil de Estilos de Vida Promotoras de Salud. Universidad de Nebraska Medical Center.

Zieve, D., \& R. Eltz, D. (2012). Previously reviewed by Fred K. Berger. A.D.A.M. Health Solutions, Ebix, Inc. 
Copyright (c) 2019 Marycruz Baéz Palmeros, Claudia Beatriz Enriquez Hernández, Edith Castellanos Contreras, Maria Esther Fragoso Terán y Javier Salazar Mendoza

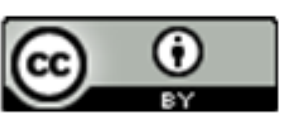

Este texto está protegido por una licencia licencia CreatriveCommons 4.0.

Usted es libre para Compartir —copiar y redistribuir el $\mathrm{m}$ aterial en cualquier medio o formato- y Adaptar el documento — remezclar, transformar y crear a partir del material- para cualquier propósito, incluso para fines com erciales, siempre que cumpla la condición de:

Atribución: Usted debe dar crédito a la obra original de manera adecuada, proporcionar un enlace a la licencia, e indicar si se han realizado cambios. Puede hacerlo en cualquier forma razonable, pero no de forma tal que sugiera que tiene el apoyo del licenciante o 10 recibe por el uso que hace de la obra.

Resumenclelicencia - Textocompletodelalicencia 